\title{
Caracterización geobotánica y validación fitosociológica de algunos sintaxones de prados salobres (all. Juncion maritimi y Plantaginion crassifoliae, class. Juncetea maritimi) de Cataluña y del Mediterráneo occidental
}

\section{Gabriel Mercadal i Corominas}

Herbari de la Universitat de Girona, Facultat de Ciències, Campus Montilivi. C \M. Aurèlia Campmany, 69. 17003 Girona, España.

\section{Correspondencia}

Gabriel Mercadal i Corominas

e-mail: g.mercadal.corominas@gmail.com

Recibido: 15 junio 2020

Aceptado: 16 septiembre 2020

Publicado on-line: 8 octubre 2020

Editado por: Andrés V. Pérez Latorre

\begin{abstract}
Resumen
Presentamos sintéticamente una parte de los resultados fitogeográficos descritos en la tesis doctoral del autor. En este caso, caracterizamos y validamos algunos sintaxones de prados salobres de las alianzas Juncion maritimi y Plantaginion crassifoliae (class. Juncetea maritimi) del nordeste de Cataluña (incl. NE de España y SE de Francia) y del Mediterráneo occidental. En total, estudiamos geobotánicamente 15 sintaxones a partir de tablas sintéticas y análisis factoriales de correspondencias publicados previamente en la tesis: 1 clase (Juncetea maritimi), 1 orden (Juncetalia maritimi), 2 alianzas (Juncion maritimi; Plantaginion crassifoliae), 2 subalianzas (Eu-Plantaginenion crassifoliae; Limonio virgatiPlantaginenion crassifoliae nova), 5 asociaciones y 4 subasociaciones (Oenantho fistulosae-Lotetum glabri nova; Schoeno nigricantis-Plantaginetum crassifoliae; Orchido palustris-Festucetum arundinaceae nova; Limonio virgatiPlantaginetum crassifoliae nova subass. typicum, subass. inuletosum mediterraneae nova, subass. helichrysetosum stoechadis nova, subass. caricetosum divisae nova; Trifolio squamosi-Plantaginetum crassifoliae nova).
\end{abstract}

Palabras clave: Cataluña, Mediterráneo occidental, caracterización geobotánica, validación fitosociológica, prados salobres, Juncion maritimi, Plantaginion crassifoliae.

\section{Résumé}

Caractérisation géobotanique et validation phytosociologique de certaines syntaxons des prairies saumâtres (all. Juncion maritimi et Plantaginion crassifoliae, class. Juncetea maritimi) de la Catalogne et de la Méditerranée occidentale

Nous présentons synthétiquement une partie des résultats phytogéographiques décrits dans la thèse de doctorat de l'auteur. Dans ce cas, nous caractérisons et validons certaines syntaxons des prairies saumâtres des alliances Juncion maritimi et Plantaginion crassifoliae (class. Juncetea maritimi) du nord-est de la Catalogne (comprend NE de l'Espagne et SE de la France) et de la Méditerranée occidentale. Au total, nous avons étudié 15 syntaxons géobotaniquement à partir des tableaux synthétiques et des analyses factorielles de correspondances précédemment publiées dans la thèse : 1 classe (Juncetea maritimi), 1 ordre (Juncetalia maritimi), 2 alliances (Juncion maritimi; Plantaginion crassifoliae), 2 sous-alliances (Eu-Plantaginenion crassifoliae; Limonio virgati-Plantaginenion crassifoliae nova), 5 associations et 4 sous-associations (Oenantho fistulosaeLotetum glabri nova; Schoeno nigricantis-Plantaginetum crassifoliae; Orchido palustris-Festucetum arundinaceae nova; Limonio virgati-Plantaginetum crassifoliae nova subass. typicum, subass. inuletosum mediterraneae nova, subass. helichrysetosum stoechadis nova, subass. caricetosum divisae nova; Trifolio squamosi-Plantaginetum crassifoliae nova).

Mots-clés : Catalogne, Méditerranée occidentale, caractérisation géobotanique, validation syntaxonomique, prairies saumâtres, Juncion maritimi, Plantaginion crassifoliae.

\section{Introducción}

En 2019, el autor de este artículo defendió en la Universitat de Girona su tesis doctoral sobre los prados de siega de la región mediterránea de
Cataluña (Mercadal, 2019a, 2019b, 2019c). Sin embargo, al tratarse de una monografía, los nuevos sintaxones descritos son inválidos según el artículo 1 del Código Internacional de Nomenclatura Fitosociológica (Weber et al., 2000; Theurillat et al., 
2020). Así pues, en este documento validamos y caracterizamos geobotánicamente algunos sintaxones que incluyen prados salobres regularmente segados del Mediterráneo occidental de las alianzas Juncion maritimi y Plantaginion crassifoliae (class. Juncetea maritimi), del mismo modo que ya hemos realizado para otras unidades sintaxonómicas en Mercadal (2018, 2020a, 2020b, 2021).

\section{Metodología}

Caracterizamos geobotánicamente y validamos fitosociológicamente 15 sintaxones (1 clase, 1 orden, 2 alianzas, 2 subalianzas, 5 asociaciones y 4 subasociaciones) de la clase Juncetea maritimi (all. Juncion maritimi y Plantaginion crassifoliae) estudiados, previamente, en la memoria doctoral del autor (Mercadal, 2019c: Tabla 120).

La zona de estudio se extiende por la región biogeográfica mediterránea del noreste de Cataluña (incl. NE de España y SE de Francia), concretamente por las comarcas del Rosselló y del Alt Empordà (Figs. 1a-1b). En Mercadal (2019a) se puede consultar información geobotánica detallada de los prados cartografiados en cada región. Hemos seguido el método fitosociológico tradicional de la escuela sigmatista (Braun-Blanquet, 1964, 1979), así como una línea de clasificación sintética. Nuestros inventarios los hemos comparado mediante tablas sintéticas y análisis factoriales de correspondencias con los de distintas comunidades vegetales afines del Mediterráneo occidental, especialmente las más próximas al noreste de Cataluña (Fig. 1a; Mercadal, 2019c: Tabla 120). Estos datos analíticos y otros ecológicos y corológicos los referenciamos en el artículo mediante citaciones bibliográficas directas a la tesis. Solo reproducimos algunos mapas y dos tablas que consideramos esenciales para comprender el texto (Figs. 1-3 y Tablas 1-2).

Las abreviaciones sintaxonómicas utilizadas en latín o inglés son las propuestas por Weber et al. (2000) o Mucina et al. (2016); todas ellas están detalladas en Mercadal (2019c). El resto de abreviaturas y símbolos que aparecen en el trabajo son: alt. veg., altura de la vegetación; caract., característica/-as; cobert., cobertura; cond. eléct., conductividad eléctrica; dif., especie diferencial; frag., fragmento; incl., incluye; inv., inventario; p., página; prin., principalmente; terr., territorial; \#, número (código).

Respecto a la nomenclatura de los taxones, seguimos la empleada en Mercadal (2019b) que corresponde, básicamente, a la seguida por Bolòs et al. (2005) o, en su defecto, la utilizada por Tison \& Foucault (2014) o Castroviejo (1986-2019). Excepcionalmente, hemos seguido otras obras, las cuales están indicadas en Mercadal (2019b: Tabla 3). Así pues, solo indicamos la autoría de los taxones usados por primera vez en el apartado de especies características (o en su defecto, diagnósticas o diferenciales) de cada sintàxon caracterizado.
Al final del trabajo, se aporta el esquema sintaxonómico de las unidades fitosociológicas estudiadas, así como el nombre completo y autoría del resto de sintaxones mencionados en el texto.

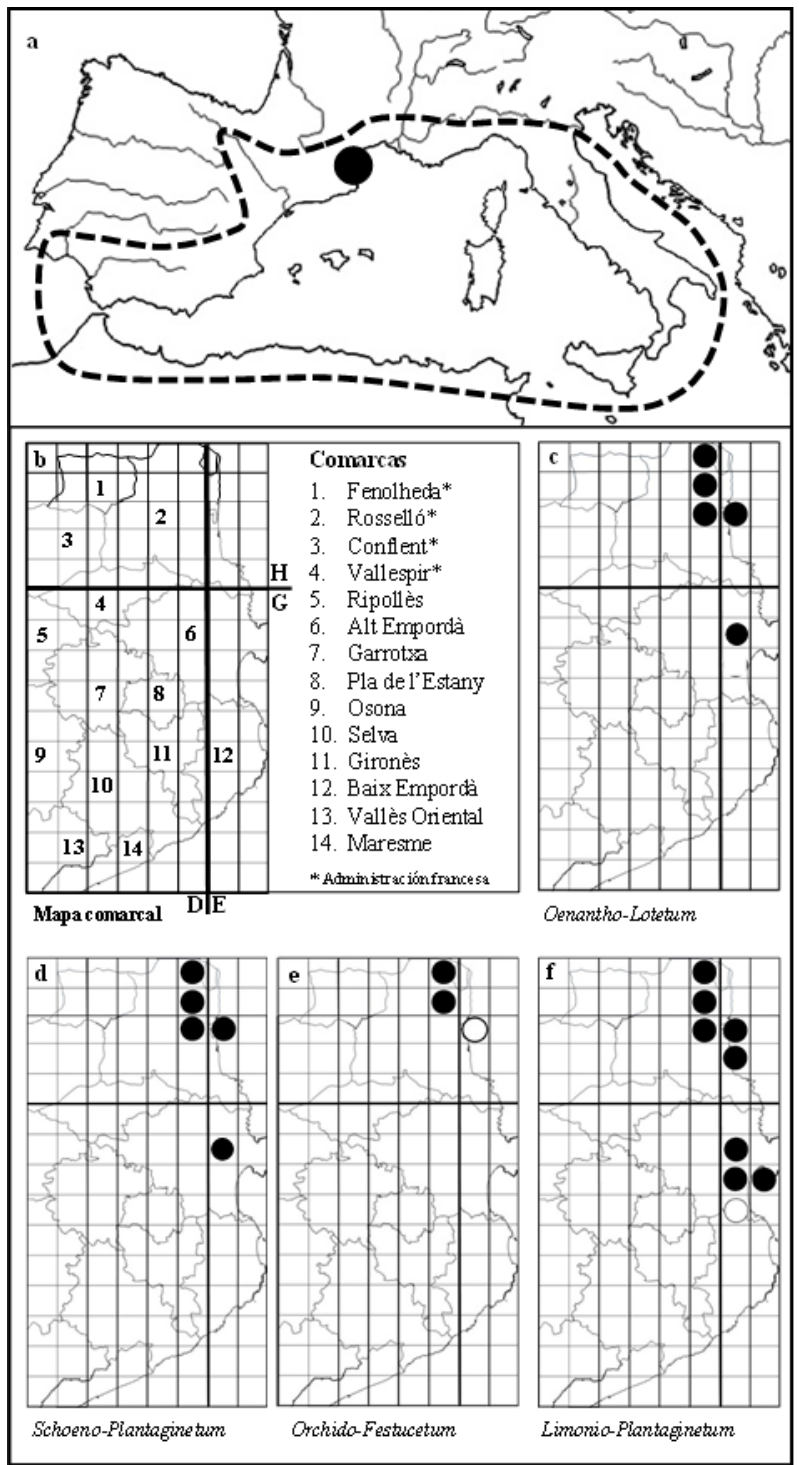

Figura 1. Localización de la zona de estudio (incl. NE de España y SE de Francia) en el ámbito del Mediterráneo occidental (a-b) y distribución de algunos sintaxones en el mapa comarcal del nordeste de Cataluña (c-f). Datos propios $(\bullet)$, datos bibliográficos $(\mathrm{O})$. Cuadrícula de los mapas, cuadrícula UTM $(10$ x $10 \mathrm{~km})$ [zona UTM 31T]. Modificado de Mercadal (2019c).

Figure 1. Localisation de la zone d'étude (comprend NE de I'Espagne et SE de la France) dans le bassin ouest de la Méditerranée (a-b) et distribution de quelques syntaxons sur la carte régionale du nord-est de la Catalogne (c-f). Données propres (•), données bibliographiques (O). Quadrillage des cartes, quadrillage UTM $(10 \times 10 \mathrm{~km})$ [zone UTM 31T]. Modifié de Mercadal (2019c).

\section{Resultados y discusión}

1. Class. Juncetea maritimi $\mathrm{Br}$--Bl. ex $\mathrm{Br}$.-Bl., Roussine et Nègre 1952 [cf. Mercadal, 2019c: 485] Sinónimos: Juncetea maritimi Br.-Bl. 1931 (phantom); Astero-Salicornietea Westhoff et al. 1942 
nom. inval. (Art. 3b); Puccinellietea phryganodisHadac 1946 (phantom); Juncetea maritimi Br.-Bl. 1950 nom. inval. (Art. 2b); Juncetea maritimi Br.-Bl. 1951 nom. inval. (Art. 2b); Juncetea maritimi Tx. et Oberd. 1958 nom. illeg. (Art. 31); Asteretea tripolii Westhoff et Beeftink in Beeftink 1962.

Holotypus: Juncetalia maritimi Br.-BI. ex Horvatić 1934; designado en Braun-Blanquet et al. (1952) (Art. 18a) [sub Juncetalia maritimi Br.-BI. 1931].

Fisionomía y composición florística: juncales y prados húmedos densos, dominados por hemicriptófitas resistentes a suelos salinos o salobres de distribución, principalmente, pluriregional: Juncus maritimus, J. acutus, J. compressus subsp. gerardi, Carex divisa, Schoenus nigricans, Lotus glaber, Trifolium squamosum, Dorycnium pentaphyllum subsp. gracile, Sonchus maritimus subsp. maritimus, Scorzonera laciniata, Elymus athericus, Hordeum secalinum, Puccinellia festuciformis, Plantago crassifolia, Linum maritimum, Centaurium pulchellum subsp. tenuiflorum...

Especies características en el Mediterráneo occidental: Juncus maritimus Lam., Alopecurus bulbosus Gouan, Apium graveolens L., Aster tripolium L., Centaurium spicatum (L.) Fritsch ex Janch., Spartina patens (Aiton) Muhl. [incl. S. versicolor E. Fabre].

Distribución: atlántico-mediterránea, principalmente por el litoral europeo, desde el sur de la costa islandesa y de la escandinava hasta la península ibérica y Grecia; y desde Galicia a Turquía. A veces, también penetra hacia el interior del continente (España, Bulgaria, Rumania, Ucrania, Macedonia, Austria...). En el norte de África, también se extiende por Marruecos (Taleb \& Fennane, 2019) y Argelia (Simonneau, 1952) (Fig. 2).

Ecología: terrenos salobres o salinos, habitualmente carbonatados, en saladares, marismas o pastos cerca del mar, que se inundan temporalmente durante los períodos lluviosos. Antiguamente, estas áreas eran regularmente pastadas y/o segadas para obtener forraje o lecho para el ganado. Hoy en día, muchas zonas han sido abandonadas y se han transformado en juncales, a menudo, densos $e$ impenetrables.

Variabilidad: Mucina et al. (2016) le adscriben cuatro órdenes en Europa. En la zona de estudio, solo se desarrolla el tipo: Juncetalia maritimi [\#1.1].

1.1. Ord. Juncetalia maritimi Br.-BI. ex Horvatić 1934 [cf. Mercadal, 2019c: 488]

Sinónimos: Juncetalia maritimi Br.-BI. 1931 nom. inval. (Art. 2b); Juncetalia maritimi Br.-Bl. 1932 nom. inval. (Art. 2b); Juncetalia maritimi Br.-Bl. 1939 nom. illeg. (Art. 31); Juncetalia maritimi Molinier et Tallon 1950 nom. illeg. (Art. 31); Juncetalia maritimi Br.-Bl. 1950 nom. inval. (Art. 2b); Juncetalia maritimi Br.-Bl. 1951 nom. inval. (Art. 2b); Juncetalia maritimi Br.-Bl. ex Br.-Bl. et al. 1952 nom. illeg. (Art. 31); Caricetalia extensae Pignatti 1953; Coeno-Halojuncetalia Chapman 1954 nom. inval. (Art. 2b); Juncetalia maritimi Br.-Bl. ex Tx. et Oberd. 1958 nom illeg. (Art. 31); Coeno-Juncetalia Chapman 1959 nom. inval.
(Art. 2b); Carici-Juncetalia gerardi (Pignatti) Passarge 1978 nom. illeg. (Art. 29).

Holotypus: Juncion maritimi Br.-BI. ex Horvatić 1934; designado en Horvatić (1934).

Fisionomía, composición florística y ecología: ídem de la clase Juncetea maritimi.

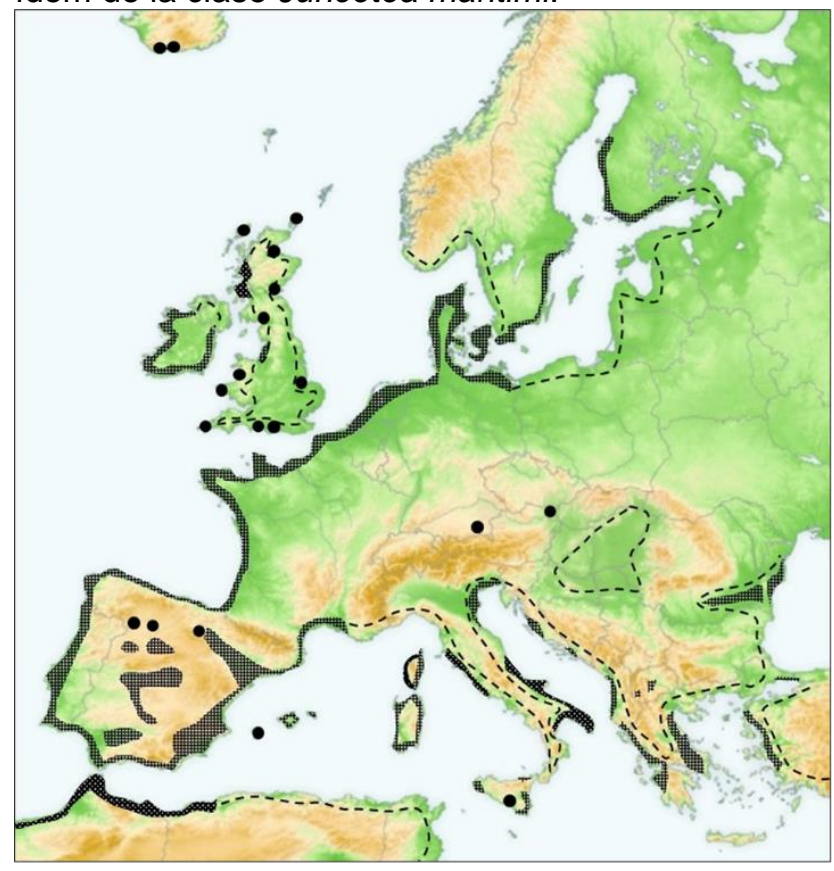

Figura 2. Distribución de la clase Juncetea maritimi en Europa y el norte de África. Línea discontinua, área potencial de la clase (Mercadal, 2019c: Fig. 210).

Figure 2. Répartition de la classe Juncetea maritimi en Europe et en Afrique du Nord. Ligne pointillée, zone potentielle de la classe (Mercadal, 2019c : Fig. 210).

Especies características en el Mediterráneo occidental: Juncus acutus L, Sonchus maritimus L. subsp. maritimus, Orchis palustris Jacq., $O$. $\times$ Iloydiana Rouy, Tetragonolobus maritimus L. (Roth), Carex extensa Gooden., Elymus athericus (Link) Kerguélen, Festuca interrupta Desf.

Distribución: Braun-Blanquet et al. (1952) dan la misma distribución para la clase Juncetea maritimi que para el orden Juncetalia maritimi. Sin embargo, Mucina et al. (2016) consideran que el orden Juncetalia es exclusivo de la región mediterránea y de la termo-atlántica, y asignan varias alianzas adscritas tradicionalmente a los Juncetalia a otros órdenes de la clase Juncetea. Así pues, según estos autores, el orden Juncetalia no se desarrollaría ni en las costas árticas ni en las del norte de Europa (islas británicas incluidas).

Variabilidad: en la zona de estudio distinguimos tres alianzas, aunque en este artículo solo caracterizamos dos: Juncion maritimi [\#1.1.1], Plantaginion crassifoliae [\#1.1.2] y Trifolion maritimi (Mercadal, 2019c: 567, 2021).

1.1.1. All. Juncion maritimi Br.-Bl. ex Horvatić 1934 [cf. Mercadal, 2019c: 496]

Sinónimos: Juncion maritimi Br.-BI. 1931 nom. inval. (Art. 2b); Juncion maritimi Br.-Bl. 1932 nom. inval. (Art. 2b); Juncion maritimi Br.-BI. 1950 nom. inval. (Art. 2b); Juncion maritimi Br.-BI. 1951 nom. inval. 
(Art. 2b); Juncion maritimi Br.-Bl. ex Br.-Bl. et al. 1952 nom. illeg. (Art. 31); Tetragonolobion siliquosi Pignatti 1953; Eco-Juncion maritimi Chapman 1954 nom. inval. (Art. 2b); Juncion maritimi Chapman 1959 nom. inval. (Art. 2b); Glauco maritimae-Juncion maritimi Géhu et Géhu-Franck 1984 nom. inval. (Art. 2b, 8); Puccinellion festuciformis Géhu et Scoppola in Géhu et al. 1984; Glauco maritimae-Juncion maritimi Géhu et Géhu-Franck ex Géhu 2017. Nombres equivalentes: Apio-Juncenion maritimi Rivas Goday et Rivas-Martínez 1963 nom. inval. (Art. 2b); Soncho crassifolii-Juncenion maritimi Rivas-Martínez 1984.

Holotypus: Juncetum maritimo-acuti Horvatić 1934; designado en Horvatić (1934).

Fisionomía y composición florística: juncales y prados halófilos, de mesohigrófilos a higrófilos, de (30)40-120(160) cm de altura y con una cobertura habitual del $100 \%$. Los taxones más frecuentes son plantas propias del Juncion maritimi y de las unidades superiores (Carex divisa, Juncus compressus. subsp. gerardi, J. maritimus, Alopecurus bulbosus, Triglochin maritimum, Scorzonera parviflora, Limonium narbonense, Lotus corniculatus subsp. preslii, L. glaber, Sonchus maritimus subsp. maritimus, Orchis palustris, O. xlloydiana, Tetragonolobus maritimus...), así como algunas especies acompañantes (Phragmites australis, Agrostis stolonifera, Plantago coronopus, Festuca interrupta, F. arundinacea...) (Mercadal, 2019c: Tabla 124).

Especies características: Scorzonera laciniata L., Plantago cornuti Gouan, Juncus compressus Jacq. subsp. gerardi (Loisel.) Hartm., Carex divisa Huds., Lotus corniculatus L. subsp. preslii (Ten.) P. Fourn., Triglochin maritimum $\mathrm{L}$.

Distribución: atlántico-mediterránea, en Europa se extiende desde la costa gallega hasta Bulgaria y Rumania (eVeg, 2019; Mercadal, 2019c; Tzonev et al., 2009). Taleb \& Fennane (2019) la citan de Marruecos y Dubuis \& Simonneau (1957) de Argelia, aunque seguramente debe llegar hasta Túnez (Fig. 3a).

Ecología: terrenos salobres del litoral y del interior del continente, inundados durante los períodos lluviosos y húmedos durante el verano. Los suelos son poco salinos, sobre todo durante la primavera, y habitualmente carbonatados. En la zona de estudio, estos prados suelen ser pastados y/o segados para obtener forraje o lecho para el ganado.

Sintaxonomía y variabilidad: siguiendo el criterio sintético, no distinguimos ninguna subunidad, aunque en la costa cantábrica y en la región termo-atlántica occitana ha sido indicada la alianza Glauco maritimae-Juncion maritimi, que a nuestro entender, se debe considerar como una subalianza. Tampoco valoramos la subalianza Apio-Juncenion maritimi ni la Soncho crassifolii-Juncenion maritimi por no estar presentes en el noreste de Cataluña.

Por otro lado, según los datos de Mucina et al. (2016), la autoría de la alianza [Juncion maritimi Br.Bl. ex Br.-Bl. et al. 1952] y la lectopificación propuestas por Rivas-Martínez et al. (2011: 200) se deben considerar ilegítima y superflua respectivamente, pues existe un nombre correcto más antiguo que contiene holotipo.
En cuanto a las comunidades del Juncion maritimi, en la zona de estudio y alrededores incluimos a seis asociaciones, pero solo caracterizamos la nueva: Caricetum divisae, Junco gerardi-Triglochinetum maritimi, Spartino versicolor-Juncetum maritimi, Junco maritimi-Iridetum spuriae, Loto presliiOenanthetum fistulosae y Oenantho fistulosaeLotetum glabri nova [\#1.1.1.1].

Respecto al Loto-Oenanthetum descrito del Languedoc (Donker \& Stevelink, 1962; Foucault \& Catteau, 2012), a nuestro juicio, esta comunidad no pertenece a la alianza Ranunculo ophioglossifoliiOenanthion fistulosae, tal como proponen Foucault \& Catteau (2012), sino que se debe asignar al Juncion maritimi, como indicaron inicialmente Donker \& Stevelink (1962), debido a la abundante presencia de plantas subhalófilas propias de los Juncetea maritimi (Mercadal, 2019c: 93, Tabla 124).

\subsubsection{Ass. Oenantho fistulosae-Lotetum glabri} ass. nova hoc loco [cf. Mercadal, 2019c: 502]

Sinónimo: Oenantho fistulosae-Lotetum glabri Mercadal 2019 nom. ined. (Art. 1).

Holotypus hoc loco designatus: Mercadal (2019c), tesis doctoral inédita: p. 508, Tabla 122, inv. 1 (Rosselló, Sant Nazari, als prats de l'estany,

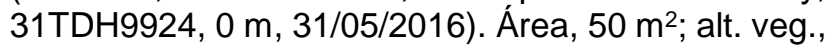
$70 \mathrm{~cm}$; cobert., 100\%: Lotus glaber 2.2, L. corniculatus subsp. preslii 2.2, L. corniculatus subsp. corniculatus var. crassifolius + , Oenanthe fistulosa 1.1, O. lachenalii 1.1, Juncus compressus subsp. gerardi 1.1, J. bufonius subsp. bufonius +, Carex divisa 3.3, C. extensa + , C. distans 1.1, C. flacca + , Alopecurus bulbosus + , Orchis palustris 1.1, 0 . laxiflora 1.1, Sonchus maritimus subsp. maritimus 1.1, Melilotus segetalis +, Apium graveolens 1.1, Iris spuria subsp. maritima 1.1, Phragmites australis 2.2, Eleocharis palustris subsp. uniglumis 1.1, Festuca arundinacea subsp. arundinacea 2.2, Trifolium fragiferum 2.2, Equisetum ramosissimum +, Ranunculus repens $1.1, R$. bulbosus,$+ R$. sardous subsp. sardous +, Vicia cracca var. argentea +, Scirpioides holoschoenus 1.1, Lycopus europaeus +, Samolus valerandi + , Taraxacum raii + .

Fisionomía y composición florística: prado halohigrófilo, de 30 a $160 \mathrm{~cm}$ de altura, y con una cobertura del $100 \%$, donde distintas plantas presentan una frecuencia y una abundancia más o menos equitativa (Lotus glaber, Oenanthe fistulosa, Festuca arundinacea, Juncus compressus subsp. gerardi, Eleocharis palustris subsp. uniglumis, Phragmites australis, Trifolium fragiferum) (Mercadal, 2019c: Tablas 122-123).

Especies diagnósticas: Lotus glaber Mill. [= Lotus corniculatus L. subsp. tenuifolius (L.) P. Fourn.], Oenanthe fistulosa L., O. lachenalii C. C. Gmel.

Distribución: litoral del Rosselló y del Empordà, entre 0 y 2 m (Fig. 1c; Mercadal, 2019c: Fig. 221). Probablemente, también penetra más al norte, en las marismas de la región de Narbona (Languedoc).

Ecología: márgenes de humedales, charcas estacionales y canales de desagüe en zonas de pastos, en ambientes regularmente segados $\mathrm{y} / \mathrm{o}$ pastados a lo largo del año. Los suelos son 
carbonatados, de reacción básica, ligeramente salinos, de textura arcillosa y ricos en materia orgánica.

Afinidades: el Oenantho-Lotetum presenta cierta afinidad con alguna comunidad de la alianza Ranunculo-Oenanthion (Baldellio ranunculoidis-
Oenanthetum fistulosae subass. caricetosum cuprinae) y, sobre todo, con algunas asociaciones del Juncion maritimi (Caricetum divisae, LotoOenanthetum); aun así, se distingue bien florística y ecológicamente, tal y como demostramos en la Tabla 1 y en Mercadal (2019c: Figs. 212, 216 y Tabla 124).

Tabla 1. Características florísticas, ecológicas y corológicas distintivas entre las asociaciones Oenantho-Lotetum, BaldellioOenanthetum subass. caricetosum, Loto-Oenanthetum y Caricetum divisae.

Tableau 1. Caractéristiques floristiques, écologiques et chorologiques distinctives entre les associations Oenantho-Lotetum, Baldellio-Oenanthetum subass. caricetosum, Loto-Oenanthetum et Caricetum divisae

\begin{tabular}{|c|c|c|c|c|}
\hline Características & $\begin{array}{l}\text { Oenantho- } \\
\text { Lotetum }\end{array}$ & $\begin{array}{l}\text { Baldellio- } \\
\text { Oenanthetum } \\
\text { caricetosum }\end{array}$ & $\begin{array}{l}\text { Loto- } \\
\text { Oenanthetum }\end{array}$ & Caricetum divisae \\
\hline $\begin{array}{l}\text { Especies } \\
\text { diferenciales entre } \\
\text { las asociaciones }\end{array}$ & $\begin{array}{l}\text { Oenanthe } \\
\text { lachenalli, Lotus } \\
\text { glaber, Phragmites } \\
\text { australis }\end{array}$ & $\begin{array}{l}\text { Baldellia } \\
\text { ranunculoides, } \\
\text { Eleocharis } \\
\text { palustris subsp. } \\
\text { palustris, Gratiola } \\
\text { officinalis, Mentha } \\
\text { pulegium }\end{array}$ & $\begin{array}{l}\text { Lotus preslii, } \\
\text { Plantago cornuti, } \\
\text { Bromus } \\
\text { commutatus, } \\
\text { Juncus fontanesii, } \\
\text { Leucojum } \\
\text { aestivum, Galium } \\
\text { palustre subsp. } \\
\text { debile }\end{array}$ & $\begin{array}{l}\text { Carex divisa, Elymus } \\
\text { athericus, Limonium } \\
\text { narbonense }\end{array}$ \\
\hline Fisionomía & $\begin{array}{l}\text { Prado higrófilo, de } \\
35-80 \mathrm{~cm}\end{array}$ & $\begin{array}{l}\text { Prado higrófilo, de } \\
35-100 \mathrm{~cm}\end{array}$ & $\begin{array}{l}\text { Prado higrófilo, de } \\
30-90 \mathrm{~cm}\end{array}$ & $\begin{array}{l}\text { Juncal mesohigrófilo, de } 20-40 \\
\mathrm{~cm}\end{array}$ \\
\hline Salinidad & $\begin{array}{l}\text { Sí (prado } \\
\text { subhalófilo) }\end{array}$ & $\begin{array}{l}\text { No (prado no } \\
\text { salino) }\end{array}$ & $\begin{array}{l}\text { Sí (prado } \\
\text { subhalófilo) }\end{array}$ & Sí (prado subhalófilo) \\
\hline $\begin{array}{l}\text { Contenido en } \\
\text { materia orgánica }\end{array}$ & Alto & Medio & Alto & Bajo \\
\hline $\begin{array}{l}\text { Contenido en } \\
\text { carbonatos }\end{array}$ & Medio-Alto & Muy bajo & Muy alto & Indiferente \\
\hline $\begin{array}{l}\text { Actividad } \\
\text { agropecuaria }\end{array}$ & $\begin{array}{l}\text { Normalmente } \\
\text { segado y/o } \\
\text { pastado }\end{array}$ & $\begin{array}{l}\text { Normalmente } \\
\text { segado y/o } \\
\text { pastado }\end{array}$ & $\begin{array}{l}\text { Normalmente } \\
\text { segado y/o } \\
\text { pastado }\end{array}$ & $\begin{array}{l}\text { Raramente segado, a veces } \\
\text { pastado }\end{array}$ \\
\hline Distribución & $\begin{array}{l}\text { Noreste de } \\
\text { Cataluña }\end{array}$ & $\begin{array}{l}\text { Noreste de } \\
\text { Cataluña }\end{array}$ & Languedoc & $\begin{array}{l}\text { Costa mediterránea catalano- } \\
\text { provenzal (incl. Baleares) }\end{array}$ \\
\hline
\end{tabular}

Por otra parte, también cabe valorar la relación fitosociológica entre el Loto-Oenanthetum y el Junco subnodulosi-Galietum debile subass. oenanthetosum fistulosae (all. Molinio-Holoschoenion), pero BraunBlanquet et al. (1952: 129) no publicaron ningún inventario de esta subasociación, ni tampoco ninguna referencia bibliográfica y, por lo tanto, desconocemos la composición florística de esta unidad. Muy probablemente, estos herbazales higrófilos de las cercanías de Montpellier correspondan al LotoOenanthetum o a un sintaxón muy próximo florísticamente.

1.1.2. All. Plantaginion crassifoliae Br.-BI. ex Tomaselli 1947 [cf. Mercadal, 2019c: 514]

Sinónimos: Plantaginion crassifoliae Br.-Bl. 1931 nom. inval. (Art. 2b); Plantaginion crassifoliae Br.-BI. 1932 nom. inval. (Art. 2b); Schoenion ferruginei Rivas Goday 1945 nom. inval. (Art. 2b); Plantaginion crassifoliae Br.-Bl. 1951 nom. illeg. (Art. 31); Plantaginion crassifoliae Br.-Bl. ex Br.-Bl. et al. 1952 nom. illeg. (Art. 31).

Lectotypus: Schoeno nigricantis-Plantaginetum crassifoliae Br.-Bl. ex Tomaselli 1947; designado en
Rivas-Martínez et al. (2011: 201) [sub SchoenoPlantaginetum Br.-Bl. in Br.-Bl. et al. 1952].

Fisionomía y composición florística: juncales y prados subhalófilos, de mesoxerófilos a mesohigrófilos, de $15-150 \mathrm{~cm}$ de altura y con una cobertura del $85-100 \%$. Los taxones más frecuentes son, básicamente, plantas propias del Plantaginion crassifoliae y de las unidades superiores (Plantago crassifolia, Juncus maritimus, J. acutus, Centaurea dracunculifolia, Lotus glaber, Sonchus maritimus, Linum maritimum, Dorycnium pentaphyllum subsp. gracile, Scirpoides holoschoenus var. australis, Orchis palustris, Tetragonolobus maritimus, Carex distans), así como algunas plantas acompañantes (Schoenus nigricans, Festuca arundinacea, Phragmites australis, Artemisia gallica...) (Mercadal, 2019c: Tabla 127).

Especies características: Plantago crassifolia Forssk., Linum maritimum L., Centaurium pulchellum (Sw.) Druce subsp. tenuiflorum (Hoffmanns. et Link) Maire, Orchis coriophora L. subsp. fragans (Pollini) Sudre, Centaurea dracunculifolia Dufour, Blackstonia perfoliata (L.) Huds. subsp. imperfoliata (L. f.) Franco et Rocha Afonso, Iris xiphium L., Scirpoides 
holoschoenus (L.) Soják var. australis (Murr.) Koch [incl. var. romanus (Murr.) Soják], Dorycnium pentaphyllum Scop. subsp. gracile (Jord.) Rouy in Rouy et Fouc.

Distribución: península balcánica (Albania), buena parte de la itálica, Córcega, Cerdeña, Sicilia, Baleares y costa valenciano-catalano-provenzal. En algunos casos, también penetra hacia el interior del continente, como en la península ibérica (Fig. 3b).

Ecología: terrenos salinos o salobres, a menudo junto al mar, pero también se puede desarrollar en el interior del continente hasta $900 \mathrm{~m}$ de altitud, sobre suelos arenosos o arcilloso-turbosos. Los prados, a veces, son pastados o segados para obtener forraje.

Sintaxonomía: alianza validada por primera vez por Tomaselli (1947: 22, Tabla I) al publicar una tabla con 20 inventarios cedidos por Braun-Blanquet del Schoeno-Plantaginetum. Posteriormente, BraunBlanquet et al. (1952) enmendaron el sintaxón al modificar y definir los taxones característicos de la unidad, así como al describir su ecología; aun así, no es necesario modificar la autoría de la unidad (Art. 47). Las autorías posteriores, como la de Br.-Bl. ex $\mathrm{Br}$.-Bl. et al. 1952 propuestas por otros autores (Rivas-Martínez et al., 2001, 2011; Bardat et al., 2004; Biondi et al., 2014; Mucina et al., 2016; eVeg, 2019) son ilegítimas (Art. 31).

Variabilidad: en el Mediterráneo occidental (Italia, Languedoc, Rosselló, islas baleares y península ibérica) distinguimos dos subalianzas (Tabla 2; Mercadal, 2019c: Tabla 127 y Figs. 226-227): suball. Eu-Plantaginenion crassifoliae [\#1.1.2.A] y suball. Limonio virgati-Plantaginenion crassifoliae nova [\#1.1.2.B].

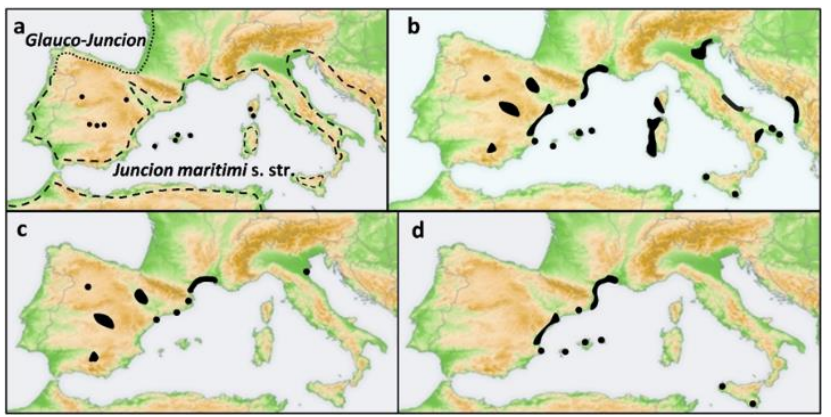

Figura 3. Distribución de los sintaxones Juncion maritimi [incl. Glauco-Juncion] (a), Plantaginion crassifoliae (b), EuPlantaginenion crassifoliae (c) y Limonio-Plantaginenion (d) en el Mediterráneo occidental. Línea discontinua, área potencial del sintaxón. Modificado de Mercadal (2019c: Figs. 215, 225, 230, 244).

Figure 3. Répartition des syntaxons Juncion maritimi [incl. Glauco-Juncion] (a), Plantaginion crassifoliae (b), EuPlantaginenion crassifoliae (c) et Limonio-Plantaginenion (d) en Méditerranée occidentale. Ligne pointillé, zone potentielle du syntaxon. Modifié de Mercadal (2019c : Figs. $215,225,230,244)$.

1.1.2.A. Suball. Eu-Plantaginenion crassifoliae [cf. Mercadal, 2019c: 524]

Fisionomía y composición florística: prados subhalófilos mesohigrófilos, de $30-150 \mathrm{~cm}$ de altura, y con una cobertura del $100 \%$. Los taxones más frecuentes son plantas de la clase Juncetea maritimi que se desarrollan en suelos húmedos: Sonchus maritimus subsp. maritimus, Dorycnium pentaphyllum subsp. gracile, Juncus maritimus, Schoenus nigricans, Orchis palustris, Linum maritimum, Carex distans, Tetragonolobus maritimus, Centaurea dracunculifolia. Plantago crassifolia, taxón característico de la alianza, también suele estar presente, pero en este caso no siempre es dominante. Por otra parte, también suele ser habitual Phragmites australis, así como varias orquidáceas: Orchis $\times$ lloydiana, O. laxiflora, O. coriophora subsp. fragans, Ophrys apifera, Serapias vomeracea... (Mercadal, 2019c: Tabla 127).

Especies características: Linum maritimum, Orchis coriophora subsp. fragans, Centaurea dracunculifolia, Iris xiphium, Dorycnium pentaphyllum subsp. gracile, Sonchus crassifolius Pourr. ex Willd. (dif. terr. Andalucía).

Distribución: costa adriática italiana septentrional, litoral catalano-provenzal e interior de la península ibérica (sierra de Granada, depresión del Ebro, submesetas norte y sur, etc.) (Fig. 3c). Probablemente, también se extiende por otros lugares del Mediterráneo occidental.

Ecología: terrenos subhalófilos, a menudo junto al mar, pero también en el interior de la península ibérica, hasta $900 \mathrm{~m}$ de altitud. Los suelos son de textura arcillosa y a menudo paraturbosos. Los prados litorales son pastados o segados para obtener forraje.

Sintaxonomía: creamos automáticamente este autónimo al dividir el Plantaginion crassifoliae en dos subunidades. El tipo nomenclatural, la autoría y la fecha son los mismos que los de la alianza.

Variabilidad: en la península ibérica y alrededores distinguimos cuatro asociaciones distintas (Mercadal, 2019c: Tabla 127 y Figs. 231-232), de las cuales solo caracterizamos las presentes en la zona de estudio: Schoeno-Plantaginetum [\#1.1.2.A.1], Centaureo dracunculifoliae-Dorycnietum gracilis, Orchido palustris-Centaureetum dracunculifoliae y Orchido palustris-Festucetum arundinaceae nova [\#1.1.2.A.2].

Cabe destacar, que Rivas-Martínez et al. (2001) adscriben el Centaureo-Dorycnietum a la subalianza Soncho-Juncenion (all. Juncion maritimi); pero, a partir de la composición florística de los inventarios estudiados, nosotros lo asignamos a la subalianza Eu-Plantaginenion crassifoliae (all. Plantaginion crassifoliae).

1.1.2.A.1. Ass. Schoeno nigricantis-Plantaginetum crassifoliae Br.-Bl. ex Tomaselli 1947 [cf. Mercadal, 2019c: 531]

Sinónimos: Schoenus nigricans-Plantago crassifoliaAssoziation Br.-Bl. 1928 nom. inval. (Art. 2b) (orig. form); Ass. à Plantago crassifolia et Schoenus nigricans Br.-BI. 1931 nom. inval. (Art. 2b) (orig. form); Schoenus nigricans-Plantago crassifolia association Br.-BI. 1932 nom. inval. (Art. 2b) (orig. form); Plantago crassifolia-Schoenus nigricans association $\mathrm{Br}$.-BI. 1932 nom. inval. (Art. 2b) (orig. form.); SchoenetoPlantaginetum crassifoliae Br.-Bl. ex Tomaselli 1947 (orig. form); Asociación Schoenus nigricans-Plantago 
crassifolia Br.-Bl. 1950 nom. inval. (Art. 2b) (orig. form); Asociación Plantago crassifolia-Schoenus nigricans Br.-Bl. 1950 nom. inval. (Art. 2b) (orig. form); Schoeneto-Plantaginetum crassifoliae Br.-Bl. 1951 nom. inval. (Art. 2b) (orig. form); SchoenetoPlantaginetum crassifoliae Br.-Bl. ex Br.-Bl. et al. 1952 nom. illeg. (Art. 31) (orig. form).

Lectotypus hoc loco designatus: Tomaselli (1947), p. 22, Tabla I, inv. 10.

Fisionomía y composición florística: prado raso mesohigrófilo, de 30-60(100) cm de altura, con una cobertura del (80)90-100\%. La asociación está dominada por Plantago crassifolia, Schoenus nigricans y Dorycnium pentaphyllum subsp. gracile. El resto de plantas comunes son, mayoritariamente, propias de la clase Juncetea maritimi: Juncus maritimus, Sonchus maritimus subsp. maritimus, Carex distans, Orchis palustris, Festuca interrupta, Elymus athericus... (Mercadal, 2019c: Tabla 127).

Especies características y diferenciales: Plantago crassifolia, Schoenus nigricans L., Scirpus cernuus Vahl (dif. terr. Languedoc), Linum maritimum, Carex extensa, Iris xiphium (dif. Languedoc), Orchis coriophora subsp. fragans (dif. terr. Languedoc), Centaurea dracunculifolia (dif. terr. Cataluña).

Distribución: litoral de Rávena (Italia), Languedoc y noreste Cataluña (Rosselló y Empordà). En el interior de la península ibérica (Braun-Blanquet \& Bolòs, 1958; Álvarez et al., 1984; Cirujano, 1981; Biurrun, 1999), la comunidad aparece muy empobrecida y se debe entender, mayoritariamente, como subasociaciones particulares 0 fragmentos de asociación (Fig. 1d; Mercadal, 2019c: Fig. 236). La asociación también se debe distribuir por otras regiones del Mediterráneo occidental.

Ecología: terrenos salobres de alrededor de marismas y en zonas deprimidas que se encharcan a menudo, encima de suelos arcillosos, básicos, más o menos carbonatados y ricos en materia orgánica. En el noreste de Cataluña, los prados normalmente son segados y/o pastados a lo largo del año.

Sintaxonomía: del mismo modo que la alianza Plantaginion crassifoliae, la asociación fue validada por primera vez por Tomaselli (1947: 22, Tabla I) al asignar los 20 inventarios cedidos por BraunBlanquet al Schoeneto-Plantaginetum crassifoliae (orig. form). Posteriormente, Braun-Blanquet et al. (1952) enmendaron el sintaxón al modificar y definir los taxones característicos de la unidad, así como al describir su ecología. La autoría Br.-Bl. in Br.-Bl. et al. 1952 sugerida por otros autores (Rivas-Martínez et al., 2001; Biondi et al., 2014; eVeg, 2019) es ilegítima (Art. 31).

Respecto al tipo nomenclatural, ni Tomaselli (1947) ni Braun-Blanquet et al. (1952) propusieron ninguno, por este motivo, lo designamos en esta publicación. Asimismo, al escoger este inventario como tipo, designamos a la vez como subasociación típica la subass. plantaginetosum crassifoliae [= subass. typicum hoc loco designatus], pues el inventario sintético utilizado para describir la subasocición (Braun-Blanquet et al., 1952: 118, inv. a) fue realizado con los inventarios publicados por Tomaselli (1947).
Variabilidad: Braun-Blanquet et al. (1952) describieron en Languedoc dos subasociaciones: la subass. plantaginetosum, la típica, y la subass. spartinetosum, una forma muy empobrecida florísticamente y que muy probablemente corresponde a una asociación distinta. En la subasociación spartinetosum faltan las especies características del Schoeno-Plantaginetum, y hay una gran abundancia de Spartina patens y Aster tripolium. Braun-Blanquet et al. (1952: 118) solo publicaron un inventario sintético de esta última unidad, el "b", realizado a partir tres inventarios de asociación. Así pues, hacen falta más inventarios para valorar la validez y la adscripción de este sintaxón.

Posteriormente, en el resto del Mediterráneo occidental, se han descrito otras subasociaciones (subass. schoenetosum, subass. dorycnietosum gracilis...) y otros sintaxones próximos (cf. p. ej.: Braun-Blanquet \& Bolòs, 1958; Cirujano, 1981; eVeg, 2019; Sciandrello et al., 2019), que hay que valorar en una próxima revisión más amplia que incluya toda la región mediterránea.

Respecto a Cataluña, por un lado, Bolòs (1962) describió la subasociación centaureo-spartinetosum, caracterizada florísticamente por Centaurea dracunculifolia y Spartina patens, pero a nuestro juicio, debe entenderse como un nombre equivalente del Orchido-Centaureetum. Por otro, Bolòs (1967) describió la subass. cyperetosum distachyi, una comunidad muy pobre florísticamente dominada por Cyperus laevigatus subsp. distachyos, y que también debe tratarse de una asociación distinta 0 de un fragmento de asociación (solo conocemos un inv.). Finalmente, Gesti \& Vilar (2002) describieron inválidamente la subasociación parapholietosum filiformis. En este caso, consideramos que este sintaxón corresponde a una nueva asociación distinta del Schoeno-Plantaginetum: Limonio-Plantaginetum [\#1.1.2.B.1]. Actualmente, pues, en la zona de estudio, no conocemos ninguna subasociación que pueda contraponerse a la subass. plantaginetosum.

1.1.2.A.2. Ass. Orchido palustris-Festucetum arundinaceae ass. nova hoc loco [cf. Mercadal, 2019c: 539]

Sinónimo: Orchido palustris-Festucetum arundinaceae Mercadal 2019 nom. ined. (Art. 1).

Holotypus hoc loco designatus: Mercadal (2019c), tesis doctoral inédita: p. 543, Tabla 131, inv. 5 (Rosselló, Salses, Garrius, 31TDH9541, 0 m, 15/05/2017). Área, $100 \mathrm{~m}^{2}$; alt. veg., $130 \mathrm{~cm}$; cobert., 100\%: Orchis palustris +, O. xlloydiana +, Ophrys apifera + , Serapias vomeracea + , Carex flacca,$+ C$. distans 1.1, Vicia cracca var. argentea 2.2, V. segetalis +, Anacamptis pyramidalis +, Centaurea dracunculifolia 1.1, Tetragonolobus maritimus 1.1, Scirpoides holoschoenus var. australis + , Sonchus maritimus subsp. maritimus 1.1, Lotus glaber 1.1, Festuca arundinacea subsp. arundinacea 3.3, Inula viscosa + , Daucus carota subsp. carota + , Holcus lanatus 2.2, Trifolium pratense 2.2, $T$. repens +, Calystegia sepium subsp. sepium 1.1, Dactylis 
glomerata subsp. glomerata 1.1, Equisetum ramosissimum subsp. ramosissimum + , Linum usitatissimum subsp. angustifolium 1.1, Picris hieracioides +, Orobanche minor +, Plantago lanceolata +, Poa trivialis subsp. trivialis 1.1, Gaudinia fragilis 2.2, Medicago sativa 1.1

Fisionomía y composición florística: prado alto y denso, de 60-150 cm de altura y con una cobertura del $100 \%$. La asociación está dominada por buenas plantas forrajeras (Festuca aundinacea, Holcus lanatus, Trifolium pratense, Tetragonolobus maritimus, Vicia cracca var. argentea) y se distingue por la presencia de taxones característicos de la subalianza Plantaginenion y de las unidades superiores: Orchis palustris, O. × lloydiana, Centaurea dracunculifolia, Carex distans, Scirpoides holoschoenus var. australis, Sonchus maritimus, Lotus glaber... (Mercadal, 2019c: Tablas 127, 131).

Especies características y diferenciales: Orchis palustris, O. xlloydiana, Serapias vomeracea (Burm.) Briq., Centaurea dracunculifolia, Ophrys apifera Huds. (dif.), Carex flacca Schreb. (dif.), Vicia cracca L. var. argentea Coss. et Germ. (dif.) [cf. Mercadal, 2019b: 113], Anacamptis pyramidalis (L.) Rich. (dif.), Vicia tetrasperma (L.) Schreb. subsp. gracilis (Loisel.) Hook. (dif.), Festuca aundinacea Schreb. subsp. arundinacea (dif.).

Distribución y ecología: márgenes de marismas salobres del litoral del Rosselló (alrededor de $0 \mathrm{~m}$ ) segados y pastados regularmente, sobre suelos limosos, ricos en materia orgánica y en carbonatos (Fig. 1e; Mercadal, 2019c: Fig. 240). Probablemente, la comunidad se extiende más hacia el norte, hasta pasado Montpellier (Languedoc).

Afinidad: el Orchido-Festucetum tiene cierta similitud florística y ecológica con el Agropyro pycnanthiTrifolietum maritimi subass. festucetosum arundinaceae (all. Trifolion maritimi), pero se distingue bien por su composición (Centaurea dracunculifolia, Serapias vomeracea, Vicia cracca var. argentea...), así como por no albergar plantas propias del Trifolion maritimi (Trifolium squamosum, Elymus athericus, E. campestris, Hordeum secalinum...). El Orchido-Festucetum representa una forma de transición entre las alianzas Plantaginion crassifoliae y Trifolion maritimi.

1.1.2.B. Suball. Limonio virgati-Plantaginenion crassifoliae suball. nova hoc loco [cf. Mercadal, 2019c: 546]

Sinónimo: Limonio virgati-Plantaginenion crassifoliae Mercadal 2019 nom. ined. (Art. 1).

Holotypus hoc loco designatus: Limonio virgatiPlantaginetum crassifoliae Gesti et Vilar ex Mercadal ass. nova.

Fisionomía y composición florística: prados subhalófilos, de mesófilos a mesoxerófilos, de 10$70(150) \mathrm{cm}$ de altura y con una cobertura del (50)80$100 \%$. La unidad se distingue florísticamente por especies de los Juncetea maritimi (Plantago crassifolia, Juncus acutus, J. maritimus, Schoenus nigricans, Inula crithmoides subsp. mediterranea, Limonium virgatum, Artemisia gallica, Phragmites australis, Parapholis filiformis), así como por la presencia de halófitos del género Limonium (cf. especies caract.) (Mercadal, 2019c: Tabla 127).

Especies características: Plantago crassifolia, Artemisia gallica Willd., Limonium virgatum (Willd.) Fourr. subsp. virgatum, L. densissimum (Pignatti) Pignatti, L. echioides (L.) Mill., L. ferulaceum (L.) Chaz., L. girardianum (Guss.) Fourr., L. bellifolium (Gouan) Dumort.

Distribución: conocemos esta unidad de Sicilia, Baleares y de la costa catalano-valencianoprovenzal, entre 0 y 5(110) $\mathrm{m}$ de altitud (Fig. 3d), aunque también se debe extender por otras áreas del Mediterráneo occidental, especialmente en el litoral.

Ecología: terrenos subhalófilos cerca del mar, habitualmente arenosos y con un contenido bajo en materia orgánica. Los prados litorales son pastados, aunque a veces, también se siegan y se obtiene un forraje de muy baja calidad.

Sintaxonomía: subalianza que incluye los inventarios adscritos al Schoeno-Plantaginetum del Mediterráneo occidental de suelos más arenosos y secos.

Variabilidad: Limonio virgati-Plantaginetum crassifoliae nova [\#1.1.2.B.1] y Trifolio squamosiPlantaginetum crassifoliae nova [\#1.1.2.B.2].

1.1.2.B.1. Ass. Limonio virgati-Plantaginetum crassifoliae Gesti et Vilar ex Mercadal ass. nova hoc loco [cf. Mercadal, 2019c: 552]

Sinónimo: Limonio virgati-Plantaginetum crassifoliae Gesti et Vilar ex Mercadal 2019 nom. ined. (Art. 1). Pseudónimo: Schoeno-Plantaginetum crassifoliae auct., non Br.-Bl. ex Tomaselli 1947. Nombre equivalente: Schoeno-Plantaginetum subass. parapholietosum filiformis Gesti et Vilar 2002 nom. inval. (Art. 5).

Holotypus hoc loco designatus: Gesti \& Vilar (2002), p. 32, Tabla 6, inv. 8.

Fisionomía y composición florística: prado-juncal normalmente de baja altura, de $10-80(150) \mathrm{cm}$, y con una cobertura del (50)80-100\%. Las plantas dominantes son Plantago crassifolia y Schoenus nigricans, la cuales suelen ir acompañadas por taxones propios de la clase Juncetea maritimi: Juncus acutus, J. maritimus, Limonium narbonense, Inula crithmoides subsp. mediterranea, Lotus glaber, Scirpoides holoschoenus var. australis, Parapholis filiformis... Sin embargo, la comunidad se caracteriza por varias plantas xerohalófilas (cf. especies caract.) (Mercadal, 2019c: Tabla 134).

Especies características: Atemisia gallica, Limonium virgatum subsp. virgatum, L. ferulaceum.

Distribución: litoral catalano-valenciano-provenzal (desde la Camarga hasta Alacant), Baleares y Sicilia (Brullo \& Furnari, 1976 [sub Schoeno-Plantaginetum p. max. p.]; Sciandrello et al., 2019 [sub SchoenoPlantaginetum]), aunque, la asociación se debe extender por otras regiones del Mediterráneo occidental (península itálica...). En la zona de estudio, la hemos observado en los Aiguamolls de l'Empordà y el llano del Rosselló (Fig. 1f; Mercadal, 2019c: Fig. 248).

Ecología: terrenos salobres litorales, alrededor de marismas, normalmente pastados (rara vez 
segados). En el Empordà, la asociación se desarrolla en suelos jóvenes ligeramente salinos (cond. eléct.: 600-1.000 $\mu \mathrm{S} / \mathrm{cm}$ ) y de pH básico (8-9). El contenido medio de materia orgánica es muy bajo $(1,0 \%)$ y el de carbonatos elevado (ca. 24,0\%). La textura puede variar entre franco-arcillosa y arenosa, así como la humedad de campo (7,0-12,4\%), la cual depende en gran medida de la fracción edáfica predominante (Porta et al., 1994; sub Schoeno-Plantaginetum).

Sintaxonomía: nueva asociación que incluye distintos inventarios adscritos previamente al Schoeno-Plantaginetum debido a una interpretación muy amplia del inventario sintético publicado por Braun-Blanquet et al. (1952: 118, inv. a), pues los inventarios de asociación de Tomaselli (1947) han pasado prácticamente desapercibidos para la mayoría de botánicos. El Schoeno-Plantaginetum típico es un prado mesohigrófilo y paraturboso, mientras que el Limonio-Plantaginetum corresponde a un prado-juncal mesófilo o mesoxerófilo de suelos arenosos y pobres en materia orgánica (Tabla 2).

Variabilidad: en el sector catalano-valenciano-balear incluimos cuatro subasociaciones ecológicas, que se distinguen por la textura edáfica y la actividad pecuaria (Mercadal, 2019c: Tabla 134 y Fig. 249): subass. typicum [\#1.1.2.B.1.1], subass. inuletosum mediterraneae nova [\#1.1.2.B.1.2], subass. helichrysetosum stoechadis nova [\#1.1.2.B.1.3] y subass. caricetosum divisae nova [\#1.1.2.B.1.4]. En el resto del mediterráneo occidental pueden existir otras subasociaciones geográficas.

Tabla 2. Características florísticas, ecológicas y corológicas distintivas entre las subalianzas Eu-Plantaginenion crassifoliae y Limonio-Plantaginenion.

Tableau 2. Caractéristiques floristiques, écologiques et chorologiques distinctives entre les sous-alliances EuPlantaginenion crassifoliae et Limonio-Plantaginenion.

\begin{tabular}{|c|c|c|}
\hline Características & Eu-Plantaginenion crassifoliae & Limonio-Plantaginenion \\
\hline Especies características & $\begin{array}{l}\text { Linum maritimum, Orchis coriophora } \\
\text { subsp. fragans, Centaurea dracunculifolia, } \\
\text { Iris xiphium, Dorycnium pentaphyllum } \\
\text { subsp. gracile, Sonchus crassifolius }\end{array}$ & $\begin{array}{l}\text { Plantago crassifolia, Artemisia gallica, } \\
\text { Limonium virgatum, L. densissimum, } L \text {. } \\
\text { echioides, L. ferulaceum, L. girardianum, } L \text {. } \\
\text { bellifolium }\end{array}$ \\
\hline $\begin{array}{l}\text { Especies diferenciales } \\
\text { entre las subalianzas }\end{array}$ & $\begin{array}{l}\text { Sonchus maritimus subsp. maritimus, } \\
\text { Orchis palustris, O. xlloydiana, Carex } \\
\text { distans, Oenanthe lachenalii, } \\
\text { Tetragonolobus maritimus, Taraxacum } \\
\text { sect. Palustria (prin. T. ciliare), Festuca } \\
\text { interrupta }\end{array}$ & $\begin{array}{l}\text { Juncus acutus subsp. acutus, Centaurium } \\
\text { maritimum, Carex divisa, Trifolium } \\
\text { campestre, T. lappaceum, T. resupinatum }\end{array}$ \\
\hline Fisionomía & $\begin{array}{l}\text { Prado subhalófilo mesohigrófilo, de 30-150 } \\
\mathrm{cm}\end{array}$ & $\begin{array}{l}\text { Prado-juncal subhalófilo de mesófilo a } \\
\text { mesoxerófilo, de } 10-70(150) \mathrm{cm}\end{array}$ \\
\hline $\begin{array}{l}\text { Textura edáfica } \\
\text { predominante }\end{array}$ & Arcillosa o limosa & Arenosa \\
\hline $\begin{array}{l}\text { Contenido en materia } \\
\text { orgánica }\end{array}$ & Alto & Bajo \\
\hline Actividad agropecuaria & Siega y/o pasto & Pasto, raramente siega \\
\hline Riqueza florística media & 14 taxones/inv. & 10 taxones/inv. \\
\hline $\begin{array}{l}\text { Distribución confirmada } \\
\text { en el Mediterráneo } \\
\text { occidental }\end{array}$ & $\begin{array}{l}\text { Costa adriática italiana septentrional, litoral } \\
\text { catalano-provenzal e interior de la } \\
\text { península ibérica (sierra de Granada, } \\
\text { depresión del Ebro, submesetas norte y } \\
\text { sur, etc.), de } 0 \text { a } 900 \mathrm{~m} \text { de altitud }\end{array}$ & $\begin{array}{l}\text { Sicilia, Baleares, costa valenciano- } \\
\text { catalano-provenzal), } 0 \text { a 5(110) } \mathrm{m} \text { de } \\
\text { altitud }\end{array}$ \\
\hline
\end{tabular}

1.1.2.B.1.1. Ass. Limonio-Plantaginetum subass. typicum [cf. Mercadal, 2019c: 558]

Basónimo: Schoeno-Plantaginetum Br.-Bl. ex Tomaselli 1947 subass. parapholietosum filiformis Gesti et Vilar 2002 p. max. p. nom. inval. (Art. 5). Sinónimo: Limonio-Plantaginetum Gesti et Vilar ex Mercadal 2019 nom. ined. (Art. 1) subass. parapholietosum filiformis Gesti et Vilar ex Mercadal 2019 nom. ined. (Art. 1).

Fisionomía y composición florística: prado bajo (20-80 cm) dominado por Plantago crassifolia y Limonium virgatum. Especies diferenciales: Artemisia gallica, Parapholis filiformis (Roth) C. E. Hubbard, Sporobolus pungens (Schreb.) Kunth, Limonium delicatulum (Girard) Kuntze, L. densissimum.
Ecología y distribución: prados con poca actividad pecuaria, situados en áreas deprimidas justo detrás de las dunas, sobre suelos arenosos y secos de la costa catalano-valenciana.

Sintaxonomía: subasociación típica que constituye una transición entre las alianzas Plantaginion crassifoliae y Limonion confusi.

1.1.2.B.1.2. Ass. Limonio-Plantaginetum subass. inuletosum mediterraneae subass. nova hoc loco [cf. Mercadal, 2019c: 559]

Sinónimo: Limonio-Plantaginetum Gesti et Vilar ex Mercadal 2019 nom. ined. (Art. 1) subass. inuletosum crithmoidis Mercadal 2019 nom. ined. (Art. 1). 
Holotypus hoc loco designatus: Farràs \& Velasco (1994), p. 214, Tabla XVII, inv. 5.

Fisionomía y composición florística: juncal bajo (10-70(100) cm), dominado por Schoenus nigricans y Plantago crassifolia. Especies diferenciales: Inula crithmoides L. subsp. mediterranea Kerguélen, Juncus acutus y J. maritimus.

Ecología y distribución: juncales situados paralelamente a la línea de la costa, detrás de las dunas más próximas al mar, sobre suelos muy arenosos de Baleares y del litoral valencianocatalano-provenzal. Probablemente se extiende en otras regiones litorales, como en Sicilia y la península itálica.

Sintaxonomía: subasociación que constituye una forma de transición entre las alianzas Plantaginion crassifoliae y Inulion crithmoidis. Los inventarios sub Schoeno-Plantaginetum de Molinier et al. (1964: 23, Beauduc, primer inv.) de Provenza y de Sciandrello et al. (2019) de Sicilia podrían adscribirse a este sintaxón.

1.1.2.B.1.3. Ass. Limonio-Plantaginetum subass. helichrysetosum stoechadis subass. nova hoc loco [cf. Mercadal, 2019c: 559]

Sinónimo: Limonio-Plantaginetum Gesti et Vilar ex Mercadal 2019 nom. ined. (Art. 1) subass. helichrysetosum stoechadis Mercadal 2019 nom. ined. (Art. 1).

Holotypus hoc loco designatus: Gesti (2006), p. 672, Tabla 81, inv. 9.

Fisionomía y composición florística: juncal alto $(70-100 \mathrm{~cm})$ dominado por Schoenus nigricans y Plantago crassifolia. Especies diferenciales: Helichrysum stoechas (L.) Moench, Scabiosa atropurpurea L., Sedum sediforme (Jacq.) Pau, Ophrys sphegodes Mill.

Ecología y distribución: juncales poco activos pecuariamente, situados en las zonas de transición entre las comunidades de detrás de las dunas y las formaciones propias de las marismas, sobre suelos arcilloso-arenosos de Cataluña.

1.1.2.B.1.4. Ass. Limonio-Plantaginetum subass. caricetosum divisae subass. nova hoc loco [cf. Mercadal, 2019c: 559]

Sinónimo: Limonio-Plantaginetum Gesti et Vilar ex Mercadal 2019 nom. ined. (Art. 1) subass. caricetosum divisae Mercadal 2019 nom. ined. (Art. 1).

Holotypus hoc loco designatus: Gesti (2006), p. 672, Tabla 81, inv. 5.

Fisionomía y composición florística: prado raso $(15-70 \mathrm{~cm})$ dominado exclusivamente por Plantago crassifolia. Especies diferenciales: Carex divisa, Plantago coronopus L., Centaurium pulchellum subsp. tenuiflorum, Gaudinia fragilis (L.) P. Beauv.

Ecología y distribución: prados con cierta actividad pecuaria, a veces, corresponden a antiguos prados de siega salobres que ahora solo son pastados, situados en áreas interiores del litoral, sobre suelos arcilloso-arenosos de la costa catalano-valenciana.
Sintaxonomía: subasociación que constituye una forma de transición entre el Limonio-Plantaginetum y el Schoeno-Plantaginetum.

1.1.2.B.2. Ass. Trifolio squamosi-Plantaginetum crassifoliae ass. nova hoc loco [cf. Mercadal, 2019c: 562]

Sinónimo: Trifolio squamosi-Plantaginetum crassifoliae Mercadal 2019 nom. ined. (Art. 1).

Holotypus hoc loco designatus: Franquesa (1995), p. 401 , Tabla 58, inv. 3.

Fisionomía y composición florística: prado de 70$120 \mathrm{~cm}$ de altura con una cobertura del 90-100\%. La asociación está dominada por Plantago crassifolia y se distingue por una composición florística muy particular, que combina especies de la clase Juncetea maritimi (Juncus acutus subsp. acutus, J. compressus subsp. gerardi, Lotus corniculatus subsp. corniculatus var. crassifolius, Trifolium squamosum, Centaurium maritimum) y taxones resistentes a suelos ligeramente salobres (Trifolium lappaceum, $T$. campestre, Serapias lingua) (Mercadal, 2019c: Tabla 136).

Especies características y diferenciales: Trifolium squamosum L., T. lappaceum L., T. campestre Schreb. in Sturm, Centaurium maritimum (L.) Fritsch, Serapias lingua L., Lotus corniculatus L. subsp. corniculatus var. crassifolius (Pers.) Ser. in DC. (dif.). Distribución: comunidad muy localizada, conocida solo de la costa norte de la península de Cap de Creus (Alt Empordà, 31TEG27), entre 70 y 110 m de altitud (Franquesa, 1995; Mercadal, 2019c: Figura 252). Muy probablemente, se trate de otra comunidad endémica de esta región litoral catalana (cf. otras asociaciones exclusivas en Franquesa 1995; Mercadal, 2019c, 2021), que, a lo sumo, se debe extender un poco más hacia el norte, en la costa de la sierra de la Albera.

Ecología: depresiones de terreno de la región metamórfica silícea (cuarcitas negras y blancas) de Cap de Creus, a menudo alrededor de humedales temporales ligeramente salinizados por las gotitas de mar transportadas por el viento.

Sintaxonomía: al tratarse de una comunidad con una ecología muy singular, su adscripción resulta difícil; a pesar de todo, con quien coincide más florísticamente es con la subalianza Limonio-Plantaginenion. Las formaciones de Cap de Creus de Plantago crassifolia junto al mar, ya no pertenecen a esta asociación, y las adscribimos al Limonio-Plantaginetum.

\section{Esquema sintaxonómico}

Los sintaxones con código numérico y en negrita los hemos caracterizados geobotánicamente; el resto, solo son mencionados en el texto.

Class. JUNCETEA MARITIMI Br.-BI. ex Br.-BI. et al. 1952 [\#1]

Ord. JunCetalia maRitıMı Br.-BI. ex Horvatić 1934 [\#1.1]

All. Juncion maritimi Br.-BI. ex Horvatić 1934 [\#1.1.1]

Ass. Junco gerardi-Triglochinetum maritimi Br.-BI. ex Br.-BI. et al. 1952

Ass. Caricetum divisae Bl.-Bl. ex Br.-Bl. et al. 1952 
Ass. Spartino versicolor-Juncetum maritimi O. Bolòs 1962

Ass. Junco maritimi-Iridetum spuriae Molinier et Tallon 1968

Ass. Loto preslii-Oenanthetum fistulosae (Donker et Stevelink 1962) de Foucault in de Foucault et Catteau 2012

Ass. Oenantho fistulosae-Lotetum glabri ass. nova [\#1.1.1.1]

All. Plantaginion crassifoliae $\mathrm{Br}$.-BI. ex Tomaselli 1947 [\#1.1.2]

Suball. Eu-Plantaginenion crassifoliae [\#1.1.2.A]

Ass. Schoeno nigricantis-Plantaginetum

crassifoliae Br.-Bl. ex Tomaselli 1947 [\#1.1.2.A.1]

subass. plantaginetosum crassifoliae $\mathrm{Br}$.-BI.

in $\mathrm{Br}$.-Bl. et al. 1952 [= subass. typicum]

subass. spartinetosum $\mathrm{Br}$.-Bl. in $\mathrm{Br}$.-Bl. et al.

1952 [debe corresponder a una ass.

particular]

subass. schoenetosum $\mathrm{Br}$.-BI. et O. Bolòs 1958

subass. cyperetosum distachyi O. Bolòs 1967 [debe corresponder a una ass. particular o a un frag. de ass.]

subass. dorycnietosum gracilis Cirujano 1981 nom ined. (Art. 1)

Ass. Centaureo dracunculifoliae-Dorycnietum gracilis Esteve Chueca et Varo 1975

Ass. Orchido palustris-Centaureetum dracunculifoliae Curcó 2001 [incl. Schoeno-Plantaginetum subass. centaureo-spartitetosum O. Bolòs 1962]

Ass. Orchido palustris-Festucetum arundinaceae ass. nova [\#1.1.2.A.2]

Suball. Limonio

crassifoliae suball. nova [\#1.1.2.B]

Ass. Limonio virgati-Plantaginetum crassifoliae

Gesti et Vilar ex Mercadal ass. nova [\#1.1.2.B.1]

subass. typicum [\#1.1.2.B.1.1]

subass. inuletosum mediterraneae subass. nova [\#1.1.2.B.1.2]

subass. helichrysetosum stoechadis

subass. nova [\#1.1.2.B.1.3]

subass. caricetosum divisae subass. nova [\#1.1.2.B.1.4]

Ass. Trifolio squamosi-Plantaginetum crassifoliae ass. nova [\#1.1.2.B.2]

\section{Otros sintaxones citados en el texto}

No se incluyen las unidades indicadas en la sinonimia de los sintaxones caracterizados geobotánicamente.

Agropyro pycnanthi-Trifolietum maritimi Br.-Bl. ex Br.$\mathrm{Bl}$. et al. 1952 subass. festucetosum arundinaceae (Molinier et Tallon 1968) Mercadal 2021; Baldellio ranunculoidis-Oenanthetum fistulosae Mercadal et al. ex Mercadal et al. in Mercadal 2018 subass. caricetosum cuprinae Mercadal et al. ex Mercadal et al. in Mercadal 2018; Inulion crithmoidis Brullo et Furnari 1988; Junco subnodulosi-Galietum debile Molinier et Tallon 1950 ex Br.-Bl. in Br.-Bl. et al. 1952 subass. oenanthetosum fistulosae $\mathrm{Br}$.-BI. in $\mathrm{Br}$.-Bl. et al. 1952 nom. inval. (Art. 2b); Limonion confusi (Br.Bl. 1933) Rivas-Martínez et Costa 1984; MolinioHoloschoenion Br.-BI. ex Tchou 1948; Ranunculo ophioglossifolii-Oenanthion fistulosae de Foucault in de Foucault et Catteau 2012; Trifolion maritimi Br.-BI. ex Br.-Bl. et al. 1952.

\section{Bibliografía}

Alvárez, M., Navarro, F., Valle, C.J., Marcos, B.; Ruiz, T. \& Santos, M.T. (1984). Vegetación de los saladares castellano-leoneses. Studia Botanica, 3: 17-62.

Bardat, J., Bioret, F., Botineau, M., Boullet, V., Delpech, R., Géhu, J.M., Haury, J., Lacoste, A., Rameau, J.C., Royer, J.M., Roux, G. \& Touffet, J. (2004). Prodrome des végétations de France. Museum national d'histoire naturelle. Paris. 170 pp.

Biondi, E., Blasi, M., Allegrezza, M., Anzellotti, I., Azzella, M.-M., Carli, E., Casavecchia, S., Copiz, R., Vico, E. del., Facioni, L., Galdenzia, D., Gasparri, R., Lasen, C., Pesaresi, S., Poldini, L., Sburlino, G., Taffetani, F., Vagge, I., Zitti, S. \& Zivkovic, L. (2014). Plant communities of Italy: The Vegetation Prodrome. Plant Biosystems, 148(4): 728-814. doi:

https://doi.org/10.1080/11263504.2014.948527.

Biurrun, I. (1999). Flora y vegetación de los humedales de Navarra. Guineana, 5: 1-338.

Bolòs, O. de. (1962). El paisaje vegetal barcelonés. Facultad de Filosofía y Letras. Universidad de Barcelona. Barcelona. 192 pp.

Bolòs, O. de. (1967). Comunidades vegetales de las comarcas próximas al litoral situadas entre los ríos Llobregat y Segura. Memorias de la Real Academia de Ciencias y Artes de Barcelona, 38(1): 1-279.

Bolòs, O. de., Vigo, J., Masalles, R.M. \& Ninot, J.M. (2005). Flora manual dels Països Catalans. (3ed.). Pòrtic. Barcelona. 1310 pp.

Braun-Blanquet, J. (1964). Pflanzensoziologie. Grundzüge der vegetationskunde. (3ed.). Springer-Verlag. Wien. $865 \mathrm{pp}$.

Braun-Blanquet, J. (1979). Fitosociología. Bases para el estudio de las comunidades vegetales. Blume. Madrid. $820 \mathrm{pp}$.

Braun-Blanquet, J. \& Bolòs, O. de. (1958). Les groupements vegetaux du bassin moyen de l'Ebre et leur dynamisme. Anales Estación Experimental Aula Dei, 5: 1-274(+49).

Braun-Blanquet, J., Roussine, N. \& Nègre, R. (1952). Les Groupements Végétaux de la France Méditerranéenne. CNRS. Paris. 297 pp.

Brullo, S. \& Furnari, F. (1976). Le associazioni vegetali degli ambienti palustri costieri della Sicilia. Noticiario della Società Italiana di Fitosociologia, 11: 1-43. 
Castroviejo, S. (1986-2019). Flora iberica: plantas vasculares de la Península Ibérica e Islas Baleares. Real Jardín Botánico-CSIC. Madrid. http://www.floraiberica.es/ [20/4/2019].

Cirujano, S. (1981). Estudio florístico, ecológico y sintaxonómico de la vegetación higrófila de la submeseta sur. Tesis doctoral. Universidad Complutense de Madrid. https://eprints.ucm.es/51912/1/5303493860.pdf [12/06/2020].

Donker, M. \& Stevelink, A. (1962). Einige Wiesenvegetationen (Gaudinio-Arrhenatheretum; Molinietum mediterraneum; Caricetum divisae) im Vistre-Tal bei Le Cailar. Meded. Landbouwh. Wageningen, 61(15): 1-32.

Dubuis, A. \& Simonneau, J. (1957). Les unites phytosociologiques des terrains sales de l'Ouest Algérien. Traveaux des sections Pedologie et Agrologie, 3: 1-23.

eVeg. (2019). Végétations de France. http://www.e-veg.net [5/12/2019].

Farràs, A. \& Velasco, E. (1994). Les comunitats vegetals de les zones humides de l'Alt Empordà. Treballs de la Institució Catalana de Història Natural, 13: 167-228.

Foucault, B. de. \& Catteau, E. (2012). Contribution au prodrome des végétations de France: les Agrostietea stoloniferae Oberd. 1983. Journal de Botanique de la Société Botanique de France, 59: 5-131.

https://www.researchgate.net/publication/305476 $140[12 / 06 / 2020]$.

Franquesa, T. (1995). El paisatge vegetal de la península del cap de Creus. Arxius de les Seccions de Ciències, CIX. Secció de Ciències Biològiques. IEC. Barcelona. 628 pp.

Gesti, J. (2006). El poblament vegetal dels aiguamolls de l'Empordà. Arxius de la seccions de ciències. Vol. CXXXVIII. IEC. Barcelona. 862 pp. https://www.researchgate.net/publication/260982 615 [12/06/2020].

Gesti, J. \& Vilar, L. (2002). La vegetació halòfila dels aiguamolls de l'Empordà. Butlletí de la Institució Catalana d'Historia Natural, 70: 21-40. http://revistes.iec.cat/index.php/BICHN/article/vie w/9018/63254 [12/06/2020].

Horvatić, S. (1934). Flora i vegetacija otoka Paga. Prirodoslovna istraživanja Kraljevine Jugoslavije, 19: 116-373.

Mercadal, G. (2018). Validació de l'associació Baldellio ranunculoidis-Oenanthetum fistulosae Mercadal, Gesti \& Vilar 2008 i de diverses subassociacions del Carici remotae-Fraxinetum oxycarpae Pedrotti 1970 corr. Pedrotti 1992. Butlletí de la Institució Catalana d'Història Natural, 82: 87-88.

https://www.researchgate.net/publication/326265 $859[12 / 06 / 2020]$.

Mercadal, G. (2019a). Els prats de dall de la terra baixa catalana. Caracterització geobotànica, valoració agroambiental i estudi de les relacions fitosociològiques entre els prats dalladors de l'Europa occidental. Volum 1: introducció $i$ cartografia de les unitats pradenques. Tesi doctoral. Universitat de Girona. 669 pp. doi: https://doi.org/10.13140/RG.2.2.24042.75200.

Mercadal, G. (2019b). Els prats de dall de la terra baixa catalana. Caracterització geobotànica, valoració agroambiental i estudi de les relacions fitosociològiques entre els prats dalladors de l'Europa occidental. Volum 2: flora. Tesi doctoral. Universitat de Girona. 881 pp. doi: https://doi.org/10.13140/RG.2.2.21460.63365.

Mercadal, G. (2019c). Els prats de dall de la terra baixa catalana. Caracterització geobotànica, valoració agroambiental $i$ estudi de les relacions fitosociològiques entre els prats dalladors de l'Europa occidental. Volum 3: vegetació i gestió pradenca. Tesi doctoral. Universitat de Girona. $756+5 \mathrm{pp}$.

https://doi.org/10.13140/RG.2.2.31802.21441.

Mercadal, G. (2020a). Caracterització geobotànica dels prats de dall mesòfils de l'associació Odontito serotini-Trifolietum pratensis $\mathrm{O}$. Bolòs et Masalles 1983 (all. Arrhenatherion elatioris Koch 1926) dels Pirineus orientals catalans. In J. Bou \& L. Vilar (Eds.), Actes del XII Col-loqui Internacional de Botànica Pirenaica-Cantàbrica (pp. 181-234). Documenta universitaria.

https://www.researchgate.net/publication/342130 $153[12 / 06 / 2020]$.

Mercadal, G. (2020b). Caracterització geobotànica i validació fitosociològica de diversos sintàxons de prats de dall higròfils (ord. Deschampsietalia cespitosae) i mesohigròils (ord. TrifolioHordeetalia) de Catalunya i de l'Europa occidental. Butlletí de la Institució Catalana d'Història Natural, 84. 163-193.

doi: https://doi.org/10.2436/20.1502.01.49.

Mercadal, G. (2021). Geobotanical characterisation of the Trifolion maritimi alliance (class. Juncetea maritimi) in Catalonia and the Western Mediterranean. Hacquetia.

doi: https://doi.org/10.2478/hacq-2020-0019.

https://ojs.zrc-sazu.si/hacquetia/article/view/8839 [12/09/2020].

Molinier, R., Viano, M., Leforestier, M. \& Devaux, J.P. (1964). Etudes phytosociologiques et écologiques en Camargue et sur le plain du Bourg. Annales de la Faculté des Sciences de Marseille, XXXVI: 1100.

Mucina, L., Bültmann, K., Dierßenm, K., Theurillat, J.P., Raus, T., Čarni, A., Šumberová, K., Willner, W., Dengler, J., Gavilán, R., Chytrý, M., Hájek, M., Di Pietro, P., laukushenko, D., Pallas, J., Daniëls, F.J.A., Bergmeier, E., Santos Guerra, A., Ermakov, N., Valachovič, M.; Schaminée, J.H.J., Lysenko, T., Diduhk, Y.P., Pignatti, S., Rodwell, J.S., Capelo, J., Weber, H.E., Solomeshch, A., Dimopoulus, P., Aguiar, C., Hennekens, S.M. \& Tichý, L. (2016). Vegetation of Europe: hierarchical floristic classification system of vascular plant, bryophyte, lichen, and algal communities. Applied Vegetation Science 19(1): 3-264. doi: https://doi.org/10.1111/avsc. 12257.

Porta, J., Castells, E., Farràs, A. \& Velasco, E. (1994). Els sòls dels aiguamolls de l'Empordà: estudi de les relacions sòl-vegetació. In J. Gosálbez, J. 
Serra \& E. Velasco (Eds.), Els sistemes naturals dels aiguamolls de l'Empordà (pp. 79-118). Treballs de la Institució Catalana de Historia Natural. Vol. 13. Barcelona.

Rivas-Martínez, S., Díaz, T.E., Penas, A. \& Fernández, F. (2011). Mapa de series, geoseries y geopermaseries de vegetación de España. Memoria del mapa de Vegetación de España, parte II. Itinera Geobotanica, 18(1): 5-424. https://floramontiberica.files.wordpress.com/2013/ 01/itinerageobotanica_181_2011.pdf.

Rivas-Martínez, S., Fernández-González, F., Loidi, J., Lousa, M. \& Penas, A. (2001). Syntaxonomical checklist of vascular plant communities of Spain and Portugal to association level. Itinera Geobotanica, 14: 5-341.

https://www.researchgate.net/publication/216034 253 [12/06/2020].

Sciandrello, S., Musarella, C.M., Puglisi, M., Spanpinato, G., Tomaselli, V. \& Minissale, P. (2019). Updated and news insights on the coastal halophilos vegetation of southeastern Sicily (Italy). Plant Sociology, 56(2): 81-98.

doi: https://doi.org/10.7338/pls2019562/06.

Simonneau, P. (1952). La végétation halophile de la plaine de Perrégaux (Oran). Gouvernement Général de l'Algérie. Direction du Service de la Colonisation et de l'Hydraulique. Alger. 268 pp.

Taleb, M.S. \& Fennane, M. (2019). Vascular plant communities of Morocco. Phytosociology, Ecology and Geography. Geobotany Studies. Springer. Cham. 161 pp.

Theurillat, J-P., Willner, W. Fernández-González, F., Bültmann, K., Čarni, A., Gigante, D.; Mucina, L. \& Weber, H.E. (2020). International Code of Phytosociological Nomenclature. 4th edition. Applied Vegetation Science.

doi: https://doi.org/10.1111/avsc.12491.

Tison, J.-M. \& Foucault, B. de. (2014). Flora Gallica. Biotope. Mèze. 1196 pp.

Tomaselli, R. (1947). Metodi di rilevamento fitosociologico in uso nella Stazione Internazionale di Geobotanica di Montpellier. Communication de la SIGMA, 95. Estratto dall' Archivio Botanico, XXIII (Terza Serie - Vol. VII. Fasc. I). S.A.C. Stab. Tip. Valbonesi. Forli. 24 pp. [= Tomaselli, R. (1947). Metodi di rilevamento fitosociologico in uso nella Stazione Internazionale di Geobotanica di Montpellier. Communication SIGMA, 95. Archivio Botanico, XXIII (1): 17-38.].

Tzonev, R.T., Dimitrov, M.A. \& Roussakova, V.H. (2009). Syntaxa according to the Braun-Blanquet approach in Bulgaria. Phytologia Balcanica, 15(2): 209-213.

Weber, H.E., Moravec, J. \& Theurillat, J.-P. (2000). International Code of Phytosociological Nomenclature. 3rd edition. Journal of Vegetation Science, 11: 739-768. doi: https://doi.org/10.2307/3236580. 